\title{
Chirurgie de l'œil par laser femtoseconde
}

\author{
Caroline HUGOUNENQ', Florent DELOISON² ${ }^{2}$ Christian RATHJEN', David TOUBOUL ${ }^{3}$ \\ 'Ziemer Ophthalmic Systems AG, Allmendstrasse 11, 2562 Port, Suisse \\ ${ }^{2}$ Centre Technologique ALPhAnov, 351 cours de la libération, 33403 Talence cedex, France \\ caroline.hugouneng@ziemergroup.com
} ${ }^{3}$ Centre Hospitalier Universitaire Pellegrin, Service d'Ophtalmologie, place Amélie Raba-Léon, 33000 Bordeaux, France

Depuis le LASIK (Laser in Situ Keratomileusis), première opération de chirurgie de l'œil réalisée à l'aide d'un laser femtoseconde, les applications rendues possibles grâce à ce type de laser ne cessent de se développer (figure 1). La sophistication des systèmes utilisés lors de ces opérations implique une collaboration étroite entre les praticiens et les constructeurs, notamment pour répondre aux inconforts et aux effets secondaires ressentis par certains patients.

\section{Applications des lasers femtoseconde en chirurgie ophtalmique}

\section{Le LASIK}

Le LASIK fut la première opération réalisée grâce à un laser femtoseconde. II s'agit d'une opération consistant à la création d'un volet cornéen d'une centaine de micromètres et que l'on soulève pour pouvoir traiter le stroma cornéen (figure 2) à l'aide d'un laser généralement excimère (souvent une machine différente) dans le but de corriger des aberrations. Le volet est ensuite replacé sans suture. L'opération totale dure quelques minutes et permet de remplacer le microkératome mécanique.

\section{Traitement de la presbytie}

La presbytie est un trouble de la vision qui rend difficile la vision de près, essentiellement dû au vieillissement du cristallin qui altère la mise au point, correspondant à la perte d'élasticité du cristallin et empêchant la vue simultanée proche et lointaine. Ce phénomène intervient donc avec le vieillissement mais généralement plus tôt que les problèmes de cataracte. Un grand nombre de traitements est disponible pour la presbytie. Le but est souvent de redonner à l'œil du patient de la profondeur de champ.

Des traitements au laser femtoseconde sont déjà proposés sur la cornée. Ils visent à augmenter le rayon de courbure de la cornée pour favoriser la mise au point de

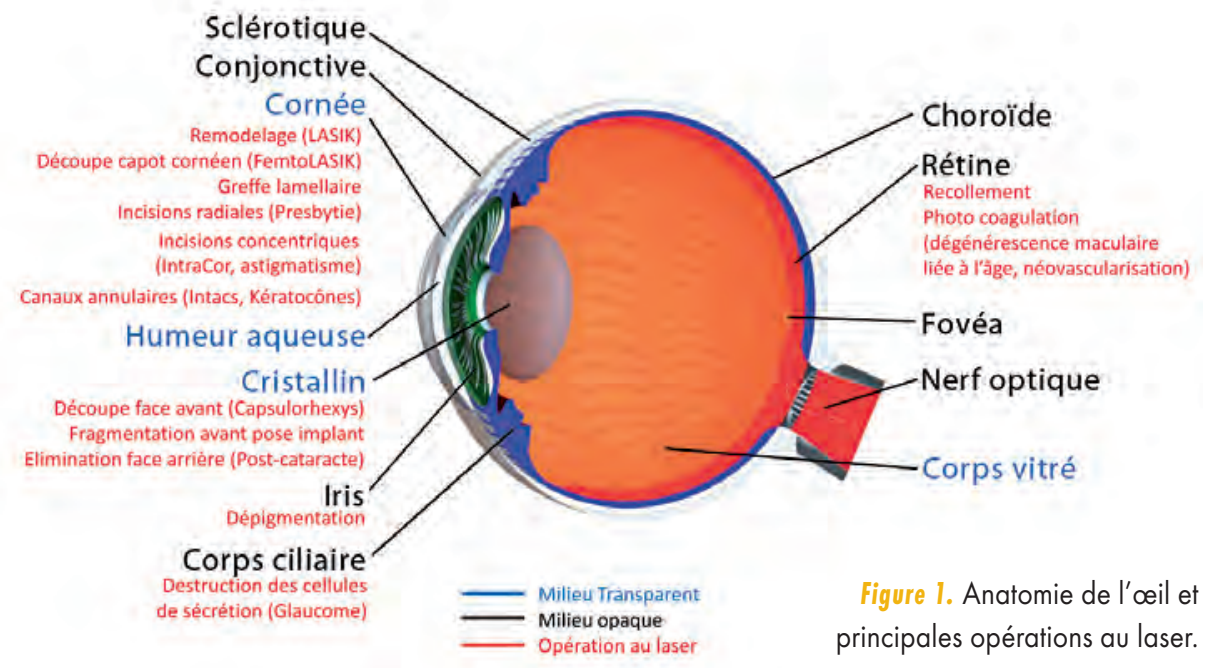

près. De plus, de nombreuses recherches sont en cours pour traiter les problèmes de presbytie au niveau du cristallin. En particulier des traitements au laser femtoseconde sur le cristallin visant à assouplir celui-ci afin de lui redonner sa souplesse sont envisagés.

\section{Quelques nouveautés dans les implants cornéens}

De nouveaux implants et logiciels associés sont développés pour le traitement de pathologies cornéennes ou de la presbytie. L'insertion des implants est grandement facilitée par l'utilisation du laser femtoseconde, qui permet d'augmenter la précision et la reproductibilité. Parfois les fabricants de tels systèmes lasers et les fabricants d'implants collaborent afin de développer une interface intuitive et parfaitement adaptée au produit.

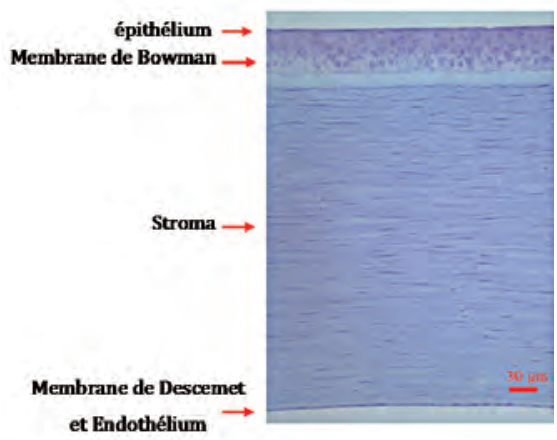

Figure 2. Anatomie de la cornée.

Implant pour le traitement de la presbytie

On peut citer par exemple l'un de ces implants qui correspond à un diaphragme biocompatible de diamètre $3,8 \mathrm{~mm}$ comprenant un trou d'un diamètre de $1,6 \mathrm{~mm}$ (implant KAMRA ${ }^{\oplus}$, Acufocus, I'un des plus 
répandus) comme présenté à la figure 3, posé uniquement sur l'un des deux yeux, pour lequel le marquage $C E$ a été obtenu récemment. D'autres implants sont déjà présents sur le marché ou en cours de développement.

L'un des avantages majeurs de tels implants est la réversibilité. L'implant est placé sous un volet classique de LASIK, cependant à une profondeur légèrement supérieure (habituellement 200 micromètres). Ainsi, il est possible de combiner au cours de la même opération un traitement de chirurgie réfractive et la pose d'un tel implant.

\section{Implant pour la kératoplastie partielle (greffe de cornée partielle)}

Parmi les implants cornéens, KeraKlear (figure 4) a développé une prothèse transparente souple en acrylique, qui se place comme un implant intrastromal pour le traitement des pathologies superficielles de la cornée. Le marquage CE a également été obtenu récemment. Cette prothèse présente de nombreux avantages, notamment le fait que l'œil ne soit pas ouvert au cours de l'opération, ce qui rend la

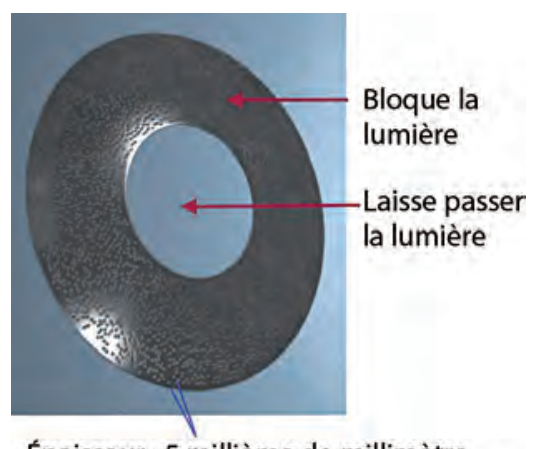

Épaisseur : 5 millième de millimètre

Figure 3. Implant Kamra [1].

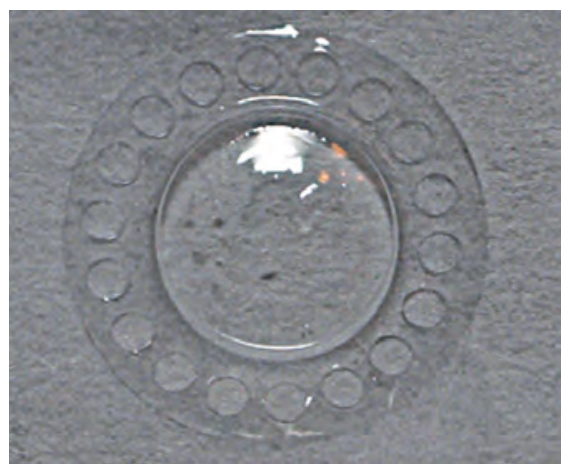

Figure 4. Implant KeraKlear (KeraMed Inc.). procédure particulièrement sûre, de plus l'opération laisse toutes les alternatives possibles pour d'autres interventions chirurgicales ultérieures. L'un des avantages majeurs d'un tel implant réside dans le fait qu'il permet de contourner la nécessité de greffer une cornée de donneur.

Certains médecins réalisent uniquement la poche intrastromale grâce à un laser femtoseconde et complètent l'ouverture d'un diamètre de 3,5 à $4 \mathrm{~mm}$ manuellement. Il est également possible de réaliser la totalité de la coupe à l'aide du laser femtoseconde et d'un logiciel sur mesure pour faciliter la précision et la sécurité de l'implantation.

\section{Traitement de la cataracte}

L'opération de la cataracte par laser est l'acte chirurgical le plus pratiqué toutes disciplines confondues avec près de 400000 interventions chaque année en France. La cataracte est aujourd'hui la première cause de cécité dans le monde du fait d'un accès difficile aux soins dans les pays les moins développés [2]. Elle touche l'ensemble de la population à partir d'un certain âge. Sa pratique est donc en constante augmentation avec le vieillissement de la population.

Récemment, la gamme des applications de chirurgie du segment antérieur de l'œil s'est étendue au cristallin au travers de la chirurgie de la cataracte. Le laser femtoseconde est alors utilisé dans les trois phases de l'opération :

- Les incisions auto-étanches sur la cornée pour faire passer les outils chirurgicaux permettant la phacoémulsification et la pose de l'implant intra-oculaire ;

- la découpe circulaire de l'enveloppe du cristallin (capsulorhexis) ;

- la fragmentation du contenu du cristallin avant aspiration et remplacement par un implant.

Plusieurs sociétés se sont lancées sur ce nouveau marché à peu près simultanément et ont obtenu ou sont en phase d'obtention des différentes autorisations (FDA, marquage $\mathrm{CE}$ ) nécessaires à leur utilisation sur l'Homme.

Les procédures d'autorisation de mise sur le marché de ces nouveaux dispositifs médicaux ont obligé les fabricants à répondre à un certain nombre de défis

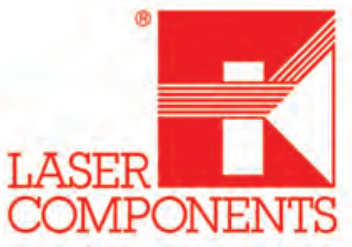

www.lasercomponents.com

\section{S'adapte} à vos besoins

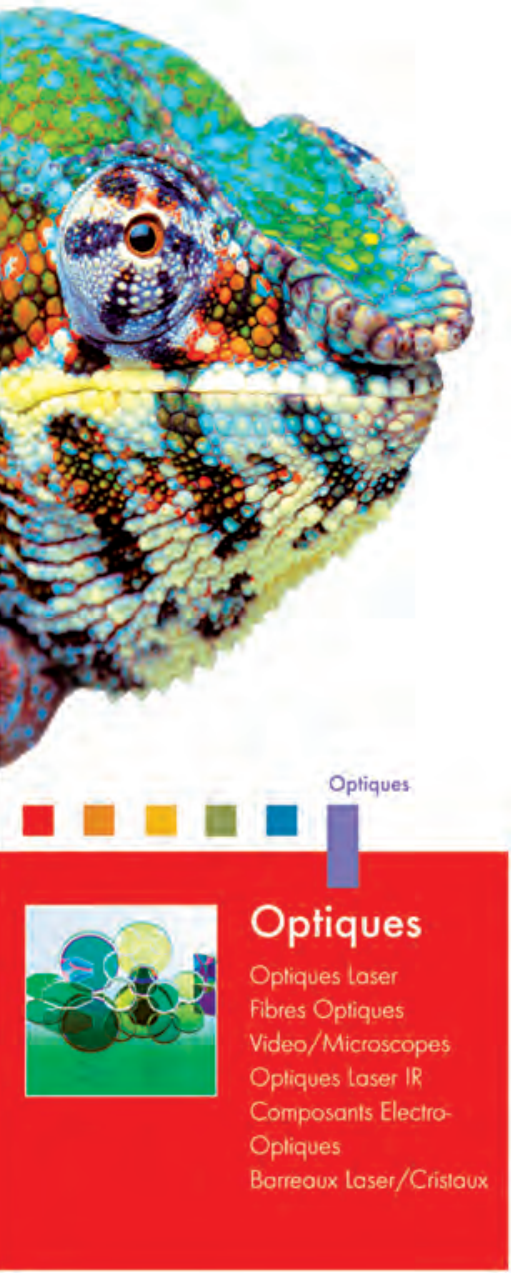

뭉 
technologiques supplémentaires qui ont dû être relevés pour parvenir à un dispositif efficace et sûr. En particulier, un système d'imagerie rapide et à haute résolution a dû être inséré pour définir précisément et en temps réel l'emplacement du centre du cristallin, son inclinaison et surtout la position de sa partie postérieure afin de ne pas risquer de la percer.

\section{Traitement du glaucome}

Le glaucome est la seconde cause de cécité dans le monde, et est une pathologie qui peut être associée à une sur pression intraoculaire entrainant la destruction progressive de la tête du nerf optique. Le traitement actuel principal consiste à abaisser la production d'humeur aqueuse (au niveau des procès ciliaires) ou à faciliter son évacuation (au niveau du trabéculum), par l'instillation de collyre ou par chirurgie. Le but de la chirurgie est de réduire la résistance du tissu au niveau du trabéculum en détruisant ou enlevant de la matière, sans endommager les tissus environnants et en évitant de provoquer une réaction cicatricielle. Les incisions réalisées par laser femtoseconde étant propres et très localisées, il s'agit d'une bonne alternative aux procédures traditionnelles, mais cette voie est actuellement à l'étape de recherche. En effet, il s'agit de traverser dans ce cas le tissu scléral, un tissu opaque et plus lâche et ayant des propriétés moins connues que le tissu cornéen, et recouvert par un tissu conjonctival responsable des processus de cicatrisation. Comme pour le traitement de la cataracte par laser femtoseconde, il est nécessaire de combiner l'outil laser à une imagerie pour positionner les incisions avec précision dans la zone d'intérêt.

Les implants comme l'iStent (Glaukos Corporation) sont aussi très utilisés pour le traitement de cette pathologie, dans le but de faciliter le drainage de l'humeur et la pose s'effectue au cours de la chirurgie manuellement ou à l'aide d'un injecteur.

\section{Modification d'indice dans les tissus} et les implants

La technologie femtoseconde permet également de modifier la densité du milieu dans lequel il est focalisé ce qui a pour conséquence de modifier locale- ment l'indice de réfraction. Ces techniques sont envisagées pour la correction de la presbytie en modifiant les indices des tissus du segment antérieur de l'œil selon un motif bien défini, ou pour corriger les aberrations résultantes dans les implants intraoculaires posés après une chirurgie de la cataracte.

Un masque de phase peut être inscrit in vivo dans le volume de l'implant comme présenté à la figure 5, afin d'induire une modification de dioptries pour pallier les incertitudes de mesure de la correction préalable à la pose de l'implant, ou les ajustements liés au positionnement de celui-ci dans l'œil.

\section{Développement et intégration des systèmes}

\section{Production}

Dès lors qu'une nouvelle machine arrive en fin de production, un grand nombre de critères - correspondant à des éléments ou à des groupes d'éléments - est vérifié pour que la machine soit validée puis envoyée aux clients. II y a par exemple toutes les vérifications concernant les caractéristiques spécifiées et la sécurité de la machine.

Parmi les nombreux critères vérifiés, il y a ceux qui concernent le faisceau laser en sortie de la machine et la transmission à travers les optiques composant la machine. On peut alors citer la durée d'impulsion ou la taille du point focal. La qualité de découpe de chaque machine est vérifiée ainsi que l'adéquation entre les paramètres de coupe choisis par le médecin (ou l'utilisateur) au niveau de l'interface-logiciel et le résultat de la coupe réelle sur l'œil subissant la coupe. Parmi ces paramètres, on trouve le diamètre et la profondeur de la coupe, l'angle sur le bord (qui est déterminant pour l'ouverture du volet en LASIK par exemple), l'orientation de la géométrie de coupe...

\section{Logiciel}

Le logiciel est un élément essentiel dans l'outil laser. Il doit permettre aux médecins de choisir les paramètres adéquats et adaptés à l'œil du patient à traiter, il
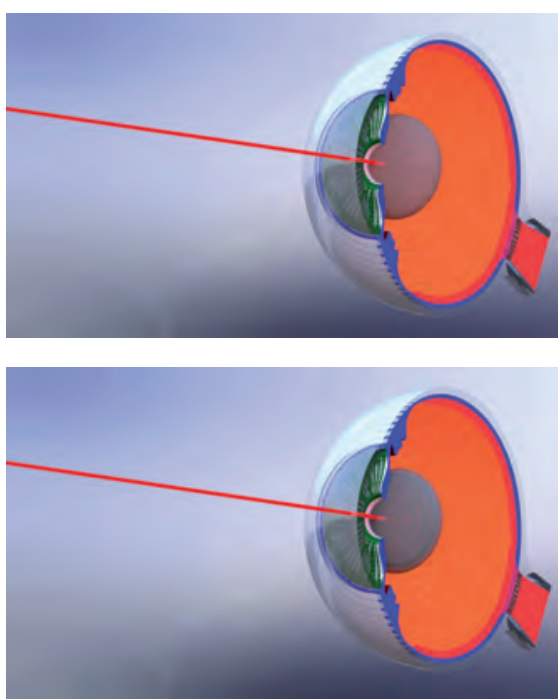

Figure 5. Représentation de l'inscription d'un masque de phase au laser dans l'œil.

doit aussi être rapide et facile à prendre en main. L'interface doit permettre aux médecins de visualiser la géométrie exigée ainsi que les paramètres choisis, elle doit permettre la visualisation correcte de l'œil du patient et le déroulement de la procédure en temps réel.

Le logiciel évolue sans cesse pour correspondre davantage aux attentes des médecins, pour ajouter de nouvelles fonctionnalités leur permettant plus de libertés et pour ajouter de nouveaux modules de découpe en fonction des développements en cours (nouveaux implants, technique améliorée...).

Par exemple, pour la pose d'un implant (comme ceux présentés dans la première partie de cet article), le médecin pourra choisir des marges par rapport aux dimensions de l'implant lui facilitant la pose, et sélectionner les paramètres les plus adéquats selon ses propres expériences.

\section{Problèmes survenus lors ou après les opérations sur patients}

L'idéal pour un fabricant est de permettre un recouvrement de la vision dès la sortie du patient de la salle d'opération. Par exemple, lors de l'opération du LASIK, certains problèmes peuvent survenir dans l'œil du patient au cours des heures et jours suivant l'opération et qui n'avaient pas été envisagés auparavant lors des tests in vitro. 
Parmi ces problèmes apparaissant suite à l'opération du LASIK et pouvant provoquer une gêne pour le patient, nous pouvons citer le syndrome de sensibilité à la lumière, la kératite lamellaire diffuse, un syndrome d'éblouissement ou encore les bulles opaques, appelées également OBL. Ces syndromes ne s'appliquent pas tous et pas de la même façon sur les différentes machines dédiées aux applications LASIK. Dans tous les cas, plus l'énergie utilisée pour l'opération est faible, moins les syndromes se produiront, jusqu'à disparaître aux énergies très basses.

Le premier syndrome (transient light sensitivity, TLS) est une sensibilité à la lumière qui apparait jusqu'à quatre semaines après l'opération et disparait généralement quelques semaines plus tard, avec ou sans un traitement stéroïdien. Ce syndrome peut être plus ou moins prononcé, provoquant un inconfort plus ou moins important pour le patient.

La kératite lamellaire diffuse (diffuse lamellar keratitis, DLK) est une réaction inflammatoire, déjà présente avec I'utilisation des microkératomes et qui n'a pas complètement disparu depuis l'utilisation des lasers femtoseconde. II s'agit de cellules migrant sous le volet en périphérie et pouvant atteindre l'axe optique (il existe plusieurs stades), donnant un aspect visuel granuleux très particulier et pouvant être douloureux. Des gouttes de cortisone sont généralement prescrites et il est parfois nécessaire de soulever à nouveau le capot cornéen pour retirer ces « infiltrats ».

Le syndrome d'éblouissement (rainbow glare) est dû au motif parfaitement régulier produit par le faisceau du laser pour générer ce volet cornéen, ce motif est similaire à un réseau de diffraction sur le stroma cornéen. Le patient est particulièrement gêné lorsqu'il regarde un point source de lumière blanche. Dans le cas où le motif est dense, cet effet diffractif et le syndrome associé disparaissent.

Le syndrome des bulles opaques (opaque bubble layer, $\mathrm{OBL}$ ) se manifeste sous la forme d'une zone blanchâtre, qui correspond à une accumulation de gaz dans la cornée du patient et qui a un effet particulièrement inesthétique. Ce gaz se résorbe dans les minutes ou heures qui suivent l'opération, mais ce phénomène peut retarder l'opération avec le laser excimère, ce qui ne satisfait pas les médecins. Les fabricants des systèmes cherchent donc à trouver le moyen d'évacuer ce gaz au cours de la coupe : certains ont recours à une géométrie particulière pour effectuer la coupe du volet afin d'éviter l'accumulation de ce gaz, d'autres ont ajouté des canaux pour permettre l'évacuation de ce gaz au cours de sa génération.

\section{Vers des applications dans d'autres spécialités?}

Cet article offre une vue d'ensemble des possibilités des lasers femtoseconde en chirurgie ophtalmique. Bien que n'étant pas exhaustif, il permet de se rendre compte de la rapidité et de l'ampleur avec lesquelles cet outil a pénétré le marché médical de l'ophtalmologie notamment grâce à une très bonne acceptance du patient et un gain en précision et en sécurité des procédures. Cette technologie reste très coûteuse ce qui limite encore son expansion vers d'autres disciplines mais il est fort probable que de prochaines évolutions et innovations continuent à répandre cette technologie dans de nombreuses applications médicales en ophtalmologie et ailleurs.

\section{Références}

[1] http://www.gatinel.com/chirurgie-refractive/ les-techniques-operatoires/implant-intra-corneenkamra-acufocus/

[2] http://www.gatinel.com/chirurgie-de-la-cataracte/

\section{Lexique}

Intrastromal : au sein du stroma.

Procés ciliaires : organes responsables de la sécrétion de l'humeur aqueuse.

Trabéculum : c'est une zone située dans l'angle irido-cornéen et responsable de la filtration de l'humeur aqueuse.

Microkératome : outil mécanique chirurgical permettant de couper un capot cornéen en laissant une charnière.

Kératocone : maladie de l'œil caractérisée par la forme de la cornée « en cône ».

Phacoémulsification : technique de fragmentation du cristallin au cours de l'opération de la cataracte.

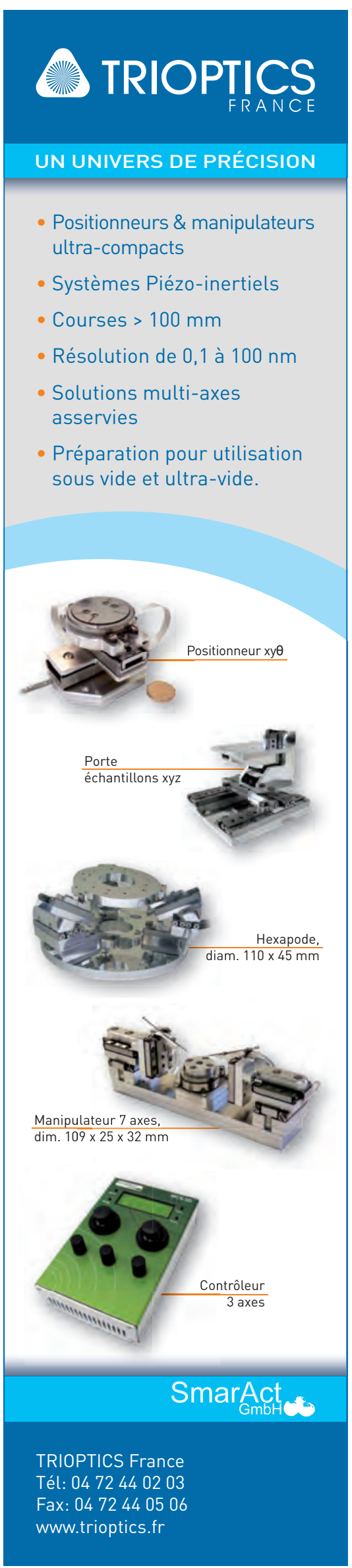

